\begin{tabular}{|c|l|}
\hline Title & A nalysis of electrocatalytic reaction schemes: distinction between rate determining and potential-determining steps \\
\hline Author(s) & Koper, Marc T. M. \\
\hline Citation & $\begin{array}{l}\text { Journal of Solid State Electrochemistry, 17(2), 339-344 } \\
\text { https://doi.org/10.1007/310008-012-1918 } \mathrm{x}\end{array}$ \\
\hline Issue Date & 2013-02-01 \\
\hline Doc URL & http://hdl.handle.net/2115/54769 \\
\hline Rights & The final publication is available at link.springer.com. \\
\hline Type & article (author version) \\
\hline File Information & JSSEMilchev-2.pdf \\
\hline
\end{tabular}

Instructions for use 


\title{
Analysis of electrocatalytic reaction schemes: distinction between rate-determining and potential-determining steps
}

\author{
Marc T.M. Koper \\ Leiden Institute of Chemistry, Leiden University, PO Box 9502, 2300 RA Leiden, The \\ Netherlands; Catalysis Research Center, Hokkaido University, Sapporo 001-0021, \\ Japan.E-mail:‥koper@chem.leidenuniv.nl
}

Dedicated to Professor Alexander Milchev on the occasion of his $70^{\text {th }}$ birthday.

\begin{abstract}
This paper discusses the concepts of rate-determining step and potential-determining step in the context of electrocatalytic reaction schemes, and illustrates how the simpler concept of potential-determining step often captures the essence of a bottleneck in a reaction scheme, and thereby provides straightforward hints for developing better catalysts.
\end{abstract}

\section{Introduction}

In starting to write a paper for an issue devoted to Alexander Milchev's $70^{\text {th }}$ birthday, I was reminded of one of Alexander's birthdays at which I was physically present, some twenty years ago. Alexander was a visiting professor at the Department of Electrochemistry of Utrecht University (the Netherlands), where I was a PhD student. For some reason, Alexander and his family had to move to another apartment. His new apartment was in a student dormitory and it wasn't very nice looking, to put it mildly. One of my colleagues and I felt embarrassed and the three of us spent a day painting the apartment to render it somewhat more habitable. At the end of a day of hard work, Alexander declared that it was time to celebrate, brought in some beer, and announced that it was his birthday. He hadn't told us anything all day long!

Since Alexander and I share a preference for simple and lucid mathematical models that highlight the essence of a physico-chemical phenomenon, in this paper I will "paint" 
some recent developments in the theoretical analysis of (electro)catalytic reaction mechanisms. In particular, I want to discuss the difference between "rate-determining step" and "potential-determining step". This seems to be a rather recent distinction, stemming from the theoretical work of Rossmeisl and Nørskov and co-workers. ${ }^{1,2}$ In this paper, I will illustrate how these two steps are not always the same, and that this observation has consequences in the search for better (electro)catalysts. In fact, I will argue, somewhat recalcitrantly, that the concept of rate-determining step is less useful in determining the optimal catalyst. Furthermore, I will show how the distinction between rate-determining and potential-determining step relates to a 10-year old discussion on the concept of the rate-determining step between Campbell ${ }^{3}$ and Dumesic ${ }^{4,5}$ in the heterogeneous catalysis literature.

\section{Model}

The concepts to be discussed will be illustrated using a well-known model for hydrogen evolution introduced more than fifty years ago by Parsons ${ }^{6,7}$ and Gerischer. ${ }^{8}$ This model has recently regained interest as a simple model explaining so-called "volcano behavior".,10,11,12 An interesting historical account of such relationships in eleectrocatalysis was presented recently by Appleby and Zagal. ${ }^{13}$ Parsons' model assumes the usual three reactions for the mechanism of hydrogen evolution:

$$
\begin{aligned}
& \mathrm{H}^{+}+*+\mathrm{e}^{-} \leftrightarrows \mathrm{H}_{\mathrm{ads}} \\
& \mathrm{H}^{+}+\mathrm{H}_{\mathrm{ads}}+\mathrm{e}^{-} \leftrightarrows \mathrm{H}_{2}+* \\
& 2 \mathrm{H}_{\mathrm{ads}} \leftrightarrows \mathrm{H}_{2}
\end{aligned}
$$

termed Volmer, Heyrovsky and Tafel reactions, respectively. Parsons expressed the rates for these reactions as a function of the electrode potential $E$, and as a function of the bond strength of $\mathrm{H}_{\mathrm{ads}}$ to the surface, $\Delta G_{H}$ (to be understood as a free energy): 


$$
\begin{aligned}
& v_{1}=k_{1}^{0}\left[H^{+}\right]\left(1-\theta_{H}\right) \exp \left(\frac{-\alpha F E-\beta \Delta G_{H}}{R T}\right) \\
& v_{-1}=k_{-1}^{0} \theta_{H} \exp \left(\frac{(1-\alpha) F E+(1-\beta) \Delta G_{H}}{R T}\right) \\
& v_{2}=k_{2}^{0}\left[H^{+}\right] \theta_{H} \exp \left(\frac{-\alpha F E-\beta \Delta G_{H}}{R T}\right) \\
& v_{-2}=k_{-2}^{0} p_{H_{2}}\left(1-\theta_{H}\right) \exp \left(\frac{(1-\alpha) E+(1-\beta) \Delta G_{H}}{R T}\right) \\
& v_{3}=k_{3}^{0} \theta_{H}^{2} \exp \left(\frac{2(1-\beta) \Delta G_{H}}{R T}\right) \\
& v_{-3}=k_{-3}^{0}\left(1-\theta_{H}\right)^{2} p_{H_{2}} \exp \left(\frac{-2 \beta \Delta G_{H}}{R T}\right)
\end{aligned}
$$

The model assumes a Butler-Volmer-type kinetics for the electrochemical steps, with $\alpha$ the corresponding transfer coefficient (that we will simply assume to be a constant equal to 0.5$)$. Variations in the binding energy of hydrogen impact on the rate constant through the Brønsted-Evans-Polanyi (BEP) coefficient $\beta .^{7}$ This coefficient is similar to the transfer coefficient and expresses a linear relation between activation energy and the reaction energy. Its applicability to heterogeneous catalysis has been discussed in detail. ${ }^{14}$ We will consider here only the rate of the hydrogen evolution reaction. Experimentally, such a reaction rate will be measured under circumstances that the back reaction can be neglected, and ideally this rate is then extrapolated to the equilibrium potential. Therefore for the remainder of the paper, we will set $v_{-2}=v_{-3}=0$. The overall rate or Faradaic current $j$ can now be calculated for the Volmer-Heyrovsky mechanism and the VolmerTafel mechanism by employing a steady-state approximation. For the Volmer-Heyrovsky mechanism, we have: 


$$
\begin{gathered}
\frac{d \theta}{d t}=0=v_{1}\left(\theta_{H, s s}^{V H}\right)-v_{-1}\left(\theta_{H, s s}^{V H}\right)-v_{2}\left(\theta_{H, s s}^{V H}\right) \Rightarrow \theta_{H, s s}^{V H}=\frac{1}{1+\frac{k_{-1}^{0}}{k_{1}^{0}\left[H^{+}\right]} \exp \left(\frac{F E+\Delta G_{H}}{R T}\right)+\frac{k_{2}^{0}}{k_{1}^{0}\left[H^{+}\right]}} \\
j=2 F v_{2}\left(\theta_{H, s s}^{V H}\right)=\frac{2 F k_{2}^{0} \exp \left(\frac{-\alpha F E-\beta \Delta G_{H}}{R T}\right)}{1+\frac{k_{-1}^{0}}{k_{1}^{0}\left[H^{+}\right]} \exp \left(\frac{F E+\Delta G_{H}}{R T}\right)+\frac{k_{2}^{0}}{k_{1}^{0}\left[H^{+}\right]}}
\end{gathered}
$$

and for the Volmer-Tafel mechanism

$$
\begin{gathered}
\frac{d \theta}{d t}=0=v_{1}\left(\theta_{H, s s}^{V T}\right)-v_{-1}\left(\theta_{H, s s}^{V T}\right)-v_{2}\left(\theta_{H, s s}^{V T}\right) ; \theta_{H, s s}^{V T}=\frac{-\left(k_{1}\left[H^{+}\right]+k_{-1}\right)+\sqrt{\left(k_{1}\left[H^{+}\right]+k_{-1}\right)^{2}+4 k_{1} k_{3}}}{2 k_{3}}(12) \\
j=2 F k_{3}^{0}\left(\theta_{H, s s}^{V T}\right)^{2} \exp \left(\frac{2(1-\beta) \Delta G_{H}}{R T}\right)
\end{gathered}
$$

where we have grouped the $k_{i}^{0}$ 's and the exponents into $k_{i}$ for notational convenience.

A plot of $\ln j$ vs. $\Delta G_{H}$ for both expressions shows that the overall rate of the reaction exhibits a maximum for $\Delta G_{H}=0$; this is the Sabatier optimum of the volcano curve. [We note that for the above expressions, the maximum rate does not occur at exactly $\Delta G_{H}=0$ because we neglected the back reactions of Eq.2 and 3]. Since the reference for $\Delta G_{H}$ is arbitrary, a more accurate definition of the top of the volcano is $d \ln j / d \Delta G_{H}=0$ (supplemented by $d^{2} \ln j / d \Delta G_{H}{ }^{2}<0$ ). Considering the importance of $\Delta G_{H}$ in optimizing the catalyst, this quantity has been called a "descriptor" by Nørskov et al. ${ }^{15}$ Descriptors can be any kind of property of the catalyst or the catalyst-reactant interaction, but it

typically represents an interaction energy. According to the Sabatier principle, ${ }^{16}$ the descriptor is the energy of interaction of the key intermediate with the catalyst.

\section{Further analysis: rate-determining step and potential-determining step, Tafel slope and "degree of rate control"}


The brief analysis above underscores shows the importance of optimizing $\Delta G_{H}$ for obtaining the best catalyst. How does this relate to the traditional concept of ratedetermining step (RDS)? Is finding the optimum of the volcano equivalent to identifying and subsequently enhancing the RDS?

Consider the two possible free energy plots illustrated in Figure 1, which would correspond to a value of $\Delta G_{H}>0$. We identify the RDS with that step in the mechanism in which the reaction passes over the highest energy in the overall energy landscape. If we consider the Volmer-Heyrovsky mechanism, the two different energy landscapes in Figure 1 correspond to (A) $k_{2} \gg k_{-1}$ and (B) $k_{2}<<k_{-1}$. In the former case, we identify $v_{1}$ as the rate-determining step, whereas in the latter case, we identify $v_{2}$ as the ratedetermining step. Conventional chemical kinetics tells us that in the former case, we must work on step 1, and in the latter case, we must work on step 2. One would want to do this by lowering the energy of the transition states of the corresponding reactions, as indicated by the red arrows. However, the easiest way to achieve such a lowering of either transition state is by lowering the energy of the intermediate state, as indicated by the green arrow, provided we believe in the abovementioned Brønsted-Evans-Polanyi relation between activation energy and reaction energy. The conclusion must be that the real problem is not in the high energies of the transition states, but in the high energy of the intermediate, or in other words, in the unfavorable thermodynamics of the first reaction. I will call this reaction the "thermodynamic bottleneck". The rate-determining step is the "kinetic bottleneck". Note that in this simple example both kinetic bottlenecks are the consequence of the existence of the thermodynamic bottleneck, and therefore identifying the thermodynamic bottleneck is a more direct strategy towards formulating a better catalyst. Further below I will give a counterexample where this conclusion does not hold, but I consider the above example more typical. 


\section{free energy}

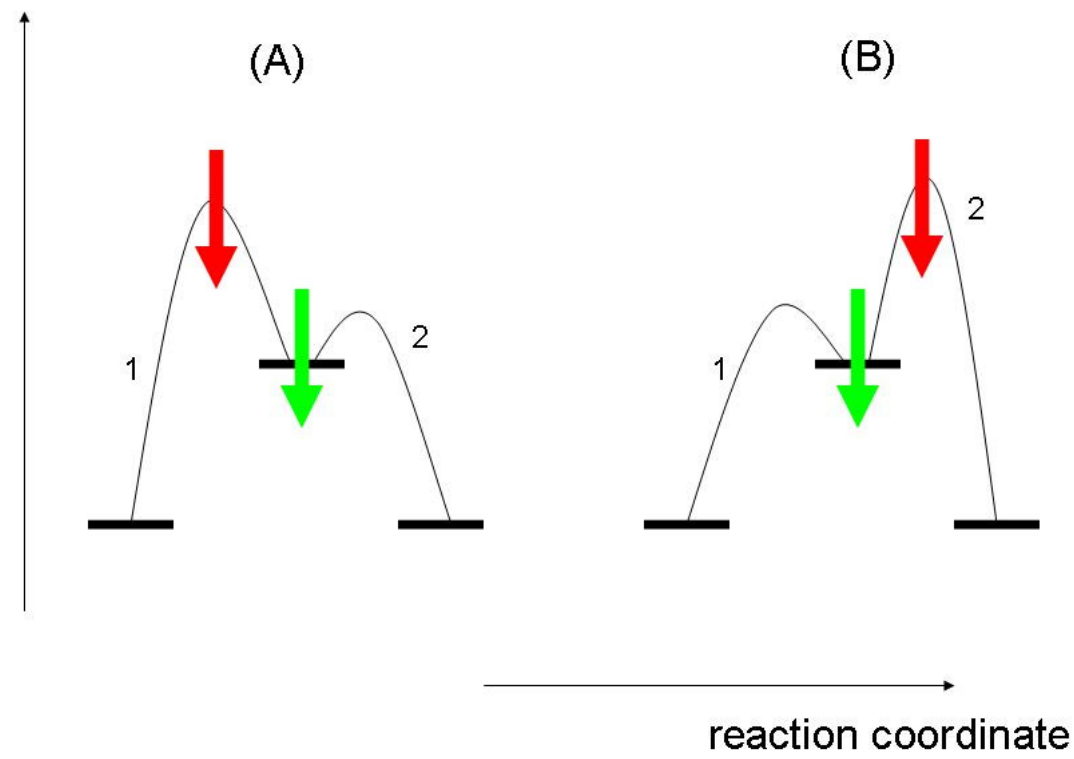

Figure 1. Two potential free energy surface for a two-step reaction. (A) step 1 is rate determining; (B) step 2 rate determining.

If the strategy is to identify the thermodynamic bottleneck rather than the kinetic bottleneck, the analysis of section 2 can be simplified considerably. The most convenient way to do this is to calculate the equilibrium potentials or, equivalently, the equilibrium constants, corresponding to each reaction step. ${ }^{1,2,17,18}$ For the Volmer-Heyrovsky mechanism, the suitably defined "standard" equilibrium potentials are given by:

$$
\begin{aligned}
& E_{1}^{e q}=-\Delta G_{H} / F \\
& E_{2}^{e q}=\Delta G_{H} / F
\end{aligned}
$$

where the energy of the $\mathrm{H}_{2}$ molecule is taken as the reference. If $\Delta G_{H}>0\left(\Delta G_{H}<0\right)$, reaction 1 (2) is thermodynamically unfavorable, with the most negative equilibrium potential. This unfavorable equilibrium potential, or unfavorable equilibrium constant, must be overcome for the reaction to proceed at a reasonable rate. Typically, a minimum overpotential of $-\left|\Delta G_{H}\right| F \mid$ must be applied for the reaction to occur. Nørskov et al. call 
this the thermodynamic overpotential, $\eta_{T}$, and the step with the most unfavorable equilibrium potential, is called the potential-determining step (PDS). Plotting $\eta_{T}$ versus $\Delta G_{H}$ gives the thermodynamic equivalent of the volcano plot (see Figure 2), with a different PDS corresponding to each leg of the plot. It is not difficult to extend such an analysis to non-electrochemical catalytic mechanisms, with equilibrium constants replacing equilibrium potentials, and to multi-step mechanisms involving multiple intermediates. $^{2,17}$ The distinction between RDS and PDS, or between thermodynamic bottleneck and kinetic bottleneck, remains the same. Also note that the thermodynamic analysis is simpler than the kinetic analysis, certainly when multi-step mechanisms are considered.

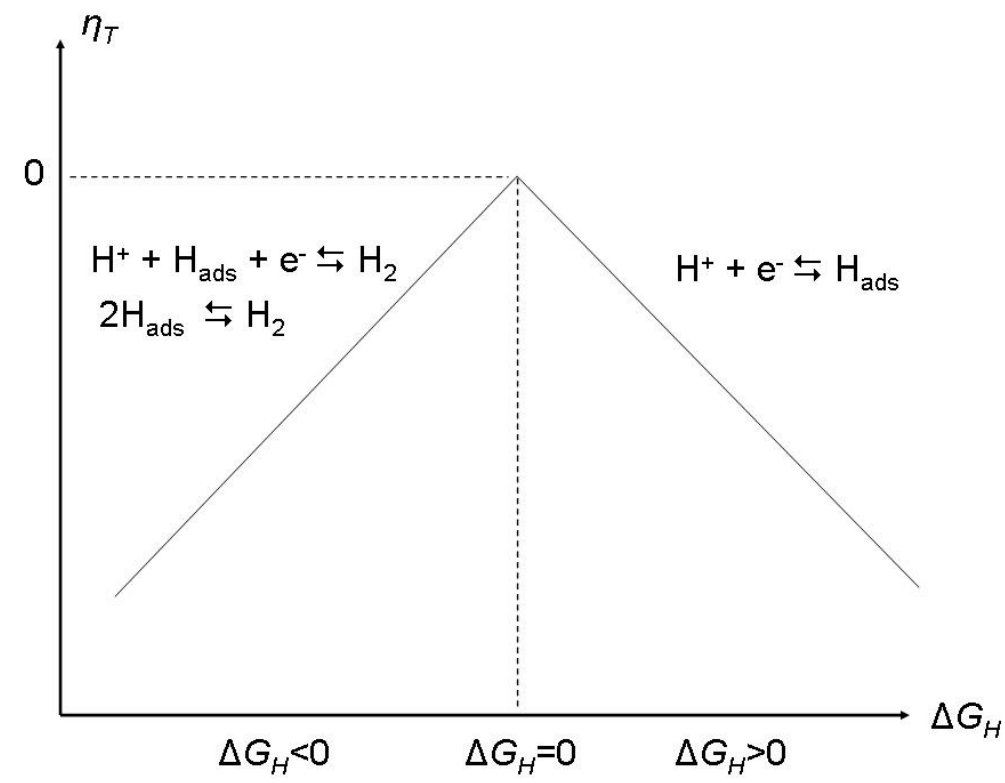

Figure 2. Thermodynamic volcano plot of the thermodynamic overpotential vs the hydrogen binding energy descriptor.

It is instructive to relate the above analysis to a standard analysis technique in electrochemistry to determine the RDS, namely Tafel slope analysis. This will also lead us to the abovementioned counterexample. Let us first consider the Volmer-Heyrovsky mechanism. The corresponding Tafel slope is defined as $d \mathrm{E} / d \log$, but for this paper I prefer to work with the following Tafel slope "TS": 


$$
T S=\left|\frac{R T}{F} \frac{d \ln |j|}{d E}\right|=\alpha+\frac{\frac{k_{-1}^{0}}{k_{1}^{0}\left[H^{+}\right]} \exp \left(\frac{F E+\Delta G_{H}}{R T}\right)}{1+\frac{k_{-1}^{0}}{k_{1}^{0}\left[H^{+}\right]} \exp \left(\frac{F E+\Delta G_{H}}{R T}\right)+\frac{k_{2}^{0}}{k_{1}^{0}\left[H^{+}\right]}}
$$

which can be considered an "effective transfer coefficient". Let me define an additional quantity that may be termed the "volcano slope" "VS":

$$
V S=R T \frac{d \ln |j|}{d \Delta G_{H}}=-\beta-\frac{\frac{k_{-1}^{0}}{k_{1}^{0}\left[H^{+}\right]} \exp \left(\frac{F E+\Delta G_{H}}{R T}\right)}{1+\frac{k_{-1}^{0}}{k_{1}^{0}\left[H^{+}\right]} \exp \left(\frac{F E+\Delta G_{H}}{R T}\right)+\frac{k_{2}^{0}}{k_{1}^{0}\left[H^{+}\right]}}
$$

Suppose that the Volmer step is the RDS. This is the case for the corresponding free energy plots shown in Figure 3(a), (d) and (f). In all cases, $k_{2}^{0} \gg>k_{-1}^{0}$, and

$$
\left|\frac{R T}{F} \frac{d \ln j}{d E}\right|=\alpha
$$

If $\alpha=0.5$, the conventional Tafel slope is ca. $119 \mathrm{mV} / \mathrm{dec}$. If we measure an experimental Tafel slope of ca. $119 \mathrm{mV} / \mathrm{dec}$, we have identified the first step as the RDS, but we do not know to which energy landscape in Figure 3 our system corresponds. Therefore, based on the Tafel analysis, the best strategy for finding the optimal catalyst is still ambiguous. 


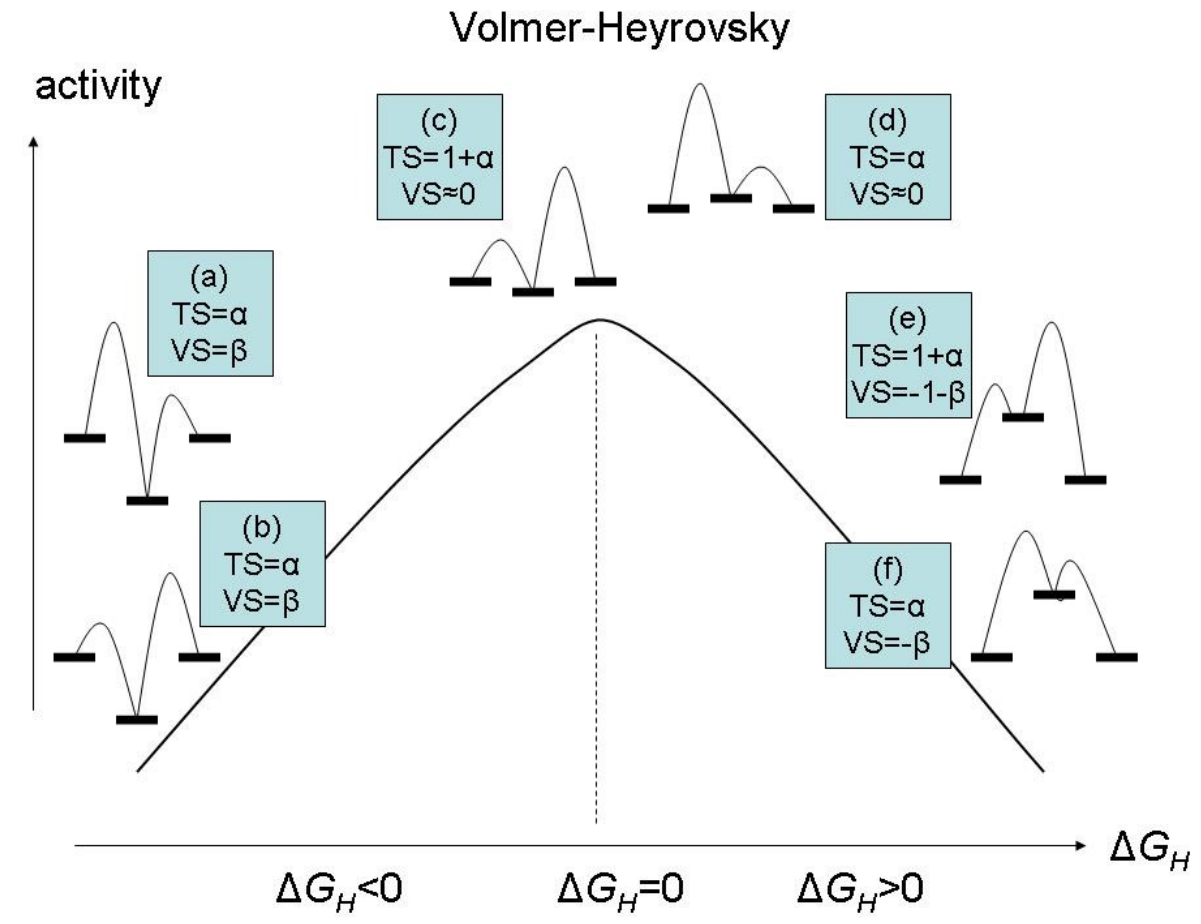

Figure 3. Volcano plot for the Volmer-Heyrovsky mechanism with typical free energy plots and corresponding Tafel slope and Volcano slope.

Combining the Tafel and the volcano analysis, let us consider the examples (a)-(f) in Figure 3 separately, as $\Delta G_{H}$ can be both positive and negative. If $\Delta G_{H}>0$, considering the situation sketched in Fig.3(f) where TS $=\alpha$, the VS $=-\beta$. If, on the other hand, we have the energy landscape sketched in Fig.3(e), the Heyrovsky reaction 2 is the RDS, $\mathrm{TS}=1+\alpha$. The volcano slope also changes, to VS $=-(1+\beta)$. Changing to the other side of the volcanic apex where $\Delta G_{H}<0$, the analysis is a bit more subtle. In case of very negative $\Delta G_{H}$, the surface is saturated with adsorbates, i.e. $\theta_{H}=1$, independent of $E$ and $\Delta G_{H}$. As a result, TS $=\alpha$ and VS $=\beta$, regardless of whether we have situation Fig.3(a) or Fig.3(b). However, note that the RDS is not the same in both cases. Close to the volcanic apex, the TS are still well-defined, but the VS are close to 0. For completeness' sake, I give the same analysis for the Volmer-Tafel analysis in Figure 4. 


\section{Volmer-Tafel}

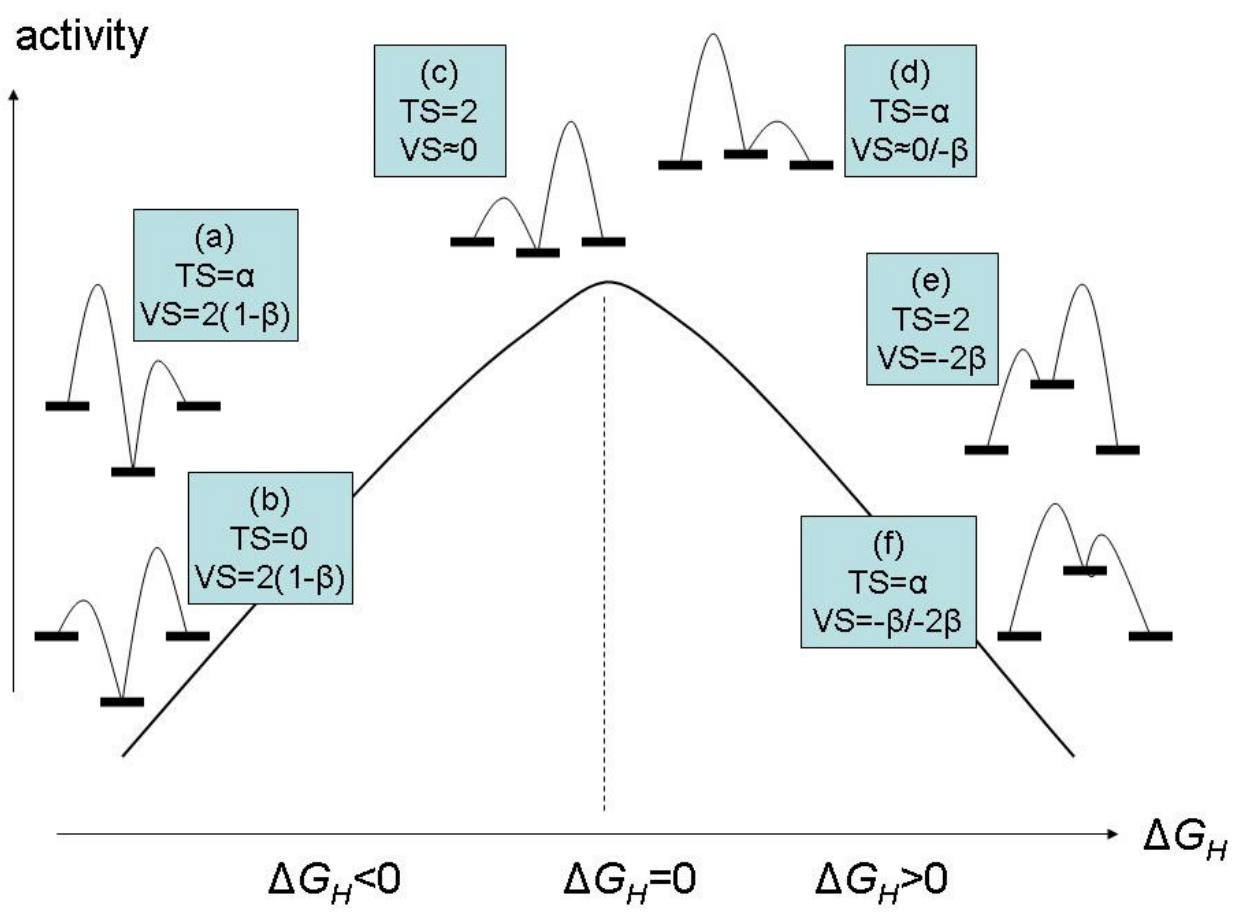

Figure 4. Volcano plot for the Volmer-Tafel mechanism with typical free energy plots and corresponding Tafel slope and Volcano slope. For large $k_{3}$, there are two volcano slopes for $\Delta G_{H}>0$. If $k_{3}>>k_{-}$, VS $=-\beta$. If $k_{3}<<k_{-1}, \mathrm{VS}=-2 \beta$. The latter happens for very large $\Delta G_{H}$.

The special cases in Figures 3 and 4 are those where the RDS and PDS are not the same. If $\Delta G_{H}>0$, reaction 1 is the PDS. However, in Fig.3(e), reaction 2 is the RDS. Nevertheless, the Sabatier strategy for developing a better catalyst, namely to strengthen the bond to the catalyst so that $\Delta G_{H}$ becomes close to zero, would work to enhance both steps. If $\Delta G_{H}<0$, reaction 2 is the PDS. In case of Fig.3(a), the reaction 1 is RDS. In this case, the Sabatier strategy, namely to weaken the bond to the surface, will make the RDS even slower, if the BEP relation applies. The general conclusion is that if the PDS or thermodynamic bottleneck is preceded by a thermodynamically favorable reaction which is, however, kinetically hindered, application of the Sabatier strategy may yield an overall slower reaction rate. This is the counterexample referred to above. If the kinetic bottleneck follows after the thermodynamic bottleneck, then typically this problem does not arise, at least not on the basis of this simple model.

The above discussion is related to similar concepts and a similar discussion that exists in the heterogeneous catalysis literature. Boudart ${ }^{19}$ and Dumesic ${ }^{4,5}$ have advocated an 
analysis of reaction schemes in terms of De Donder relations. In Dumesic' analysis, the step with the lowest reversibility is typically the rate-determining step. The reversibility of a step is equal to the exponential of De Donder's affinity of a reaction, ${ }^{20}$ where the affinity is equal to minus the change in the Gibbs free energy with respect to the extent of the reaction. A reaction step with a positive Gibbs free energy will therefore have a low affinity in De Donder's definition. It is not difficult to see that the step with the lowest reversibility is simply the PDS or thermodynamic bottleneck considered above. Campbell pointed out the limitations of the analysis based on De Donder relations, and proposed the concept of "degree of rate control".,21 In a multi-step mechanism, with overall rate $r$, the degree of rate control $X_{i}$ of step $i$ is defined as:

$$
X_{i}=\left(\frac{\partial \ln r}{\partial \ln k_{i}}\right)_{\Delta G_{i}, k_{j}}
$$

where the partial derivative is taken by holding the equilibrium constant for step $i$ constant, as well as all other reaction rate constants (as expressed by the subscript). This amounts to changing the activation energy of reaction $i$ but not its free energy. There is a relation between the degree of rate control, the Tafel slope and the volcano slope, but they do not have exactly the same meaning, as the both the TS and VS incorporate the BEP relation, whereas the definition of Eq.19 does not (for a more detailed discussion, see also ref.21). In Campbell's analysis, the step with the degree of rate control closest to unity is the RDS. In showing that this step may be different from the RDS concluded from Dumesic' analysis, Campbell ${ }^{3}$ used a hypothetical reaction scheme that corresponds exactly to the counterexample identified above: a scheme with a PDS that is preceded by a thermodynamically favorable reaction (i.e. with a high affinity) that is however kinetically hindered. Notwithstanding this counterexample, it appears that the thermodynamic or De Donder analysis is simpler, often accurate, as well as closely related to the Sabatier analysis, ${ }^{22}$ which has proven so useful in heterogeneous catalysis and electrocatalysis.

In the above analysis, I have restricted myself to simple two-step mechanisms in which there is a single clear PDS or RDS. In real mechanisms, more steps may or will be 
involved and there may not be a single PDS or RDS. This may lead to more subtle conclusions than the ones expressed above. For detailed examples, I refer to the literature. ${ }^{3,4,5,21,22}$

Finally, the distinction between RDS and PDS is also a useful way to look at the confusion that sometimes arises in the discussion of the rate-limiting step in the oxygen reduction reaction (ORR) to water. The experimentally observed Tafel slope for the ORR on a platinum electrode is typically between 60 and $120 \mathrm{mV} / \mathrm{dec}^{23,24,25}$ While I do not want to be lured into the discussion of the exact meaning of this Tafel slope, it does suggest that the first electron transfer of the overall four electron transfer reaction is either rate-determining or directly preceding the rate-determining step. On the other hand, the DFT-based thermodynamic analysis of Nørskov et al. ${ }^{1}$ has identified the removal of oxygenated species, such as $\mathrm{OH}_{\text {ads }}$, from the Pt surface, as the thermodynamic bottleneck in the reaction scheme. Therefore, many researchers have identified the reduction of $\mathrm{OH}_{\text {ads }}$ to water as the rate-determining step, i.e. the very last electron transfer step in the reaction mechanism. Strategies based on weakening the $\mathrm{OH}_{\mathrm{ads}}$ bond to the surface of the catalyst have indeed proved very successful in developing new catalysts that are more active than $\mathrm{Pt}^{26,27}$ This could be an experimental example where the RDS and PDS may indeed be different. From the modeling point-of-view, the confusion can be solved by noting that the RDS (first-electron transfer step) needs free sites on the surface in order to proceed, and those free sites are made available by working on the PDS. ${ }^{26}$ The example also illustrates two other important conclusions: (i) determination of the RDS by Tafel slope analysis does not give unambiguous hints on how to develop a better catalyst, (ii) often rate-determining steps identified in the literature are not rate-determining steps in the sense implied by Campbell's analysis, but potential-determining steps as implied by Dumesic' analysis; that is, these reaction steps are thermodynamically unfavorable, but not (necessarily) kinetically unfavorable.

\section{Conclusions}

This paper has illustrated in some detail the conceptual difference between the ratedetermining step and potential-determining step in a multi-step electrocatalytic reaction 
scheme. As the analysis based on determining the potential-determining step bears a close relationship to the Sabatier principle, identification of this step typically gives a very reliable hint on how to search for a better catalyst. The concept of rate-determining step is more general and I have discussed in which case the rate-determining step is crucially different from the potential-determining step, but also how the (experimental) determination of the rate-determining step by Tafel slope analysis does not always yield unambiguous clues for the development of a better catalyst.

\section{Acknowledgements}

I gratefully acknowledge the award of a Long-Term Fellowship of the Japanese Society for the Promotion of Science (JSPS), No. L-11527.

\section{References}

\footnotetext{
${ }^{1}$ J.K.Nørskov, J.Rossmeisl, A.Logadottir, L.Lundqvist, J.R.Kitchin, T.Bligaard, H.Jónsson, J.Phys.Chem.B 108 (2004) 17886

${ }^{2}$ J.Rossmeisl, J.Greeley, G.S.Karlberg, in Fuel Cell Catalysis: A Surface Science Approach, M.T.M.Koper (Ed.), Wiley-VCH, Hoboken NJ, USA (2009) p.57

${ }^{3}$ C.T.Campbell, J.Catal. 204 (2001) 520

${ }^{4}$ J.A.Dumesic, J.Catal. 185 (1999) 496

${ }^{5}$ J.A.Dumesic, J.Catal. 204 (2001) 525

${ }^{6}$ R.Parsons, Trans.Faraday Soc. 34 (1958) 1053

${ }^{7}$ R.Parsons, in Catalysis in Electrochemistry: From Fundamental Aspects to Strategies for Fuel Cell Development, E.Santos, W.Schmickler (Eds.), Wiley-VCH, Hoboken NJ, USA (2011) p.1

${ }^{8}$ H.Gerischer, Bull.Soc.Chim.Belg. 67 (1958) 506

9 J.K.Nørskov, T.Bligaard, A.Logadottir, J.R.Kitchin, J.G.Chen, S.Pandelov, U.Stimming, J.Electrochem.Soc. 152 (2005) J23

${ }^{10}$ W.Schmickler, S.Trasatti, J.Electrochem.Soc. 153 (2006) L31

${ }^{11}$ S.Trasatti, J.Electroanal.Chem. 39 (1977) 163

${ }^{12}$ O.A.Petrii, G.A.Tsirlina, Electrochim.Acta 39 (1994) 1739

${ }^{13}$ A.J.Appleby, J.H.Zagel, J.Solid State Electrochem. 15 (2011) 1811

${ }^{14}$ J.K.Nørskov, T.Bligaard, B.Hvolbaek, F.Abild-Petersen, I.Chorkendorff, C.H.Christensen, Chem.Soc.Rev. 37 (2008) 2163

${ }^{15}$ J.K.Nørskov, T.Bligaard, J.Rossmeisl, C.H.Christensen, Nature Chem. 1 (2009) 37

${ }^{16}$ P.Sabatier, Ber. Deutsch.Gem.Ges. 44 (1911) 1984

${ }^{17}$ M.T.M.Koper, J.Electroanal.Chem. 660 (2011) 254

${ }^{18}$ M.T.M.Koper, H.A.Heering, in Fuel Cell Science: Theory, Fundamentals, and Bio-Catalysis, A.Wieckowski, J.K.Nørskov (Eds.), Wiley-VCH, New York, 2010, p.71.

${ }^{19}$ M.Boudart, Ind.Eng.Chem.Fundam. 25 (1986) 70

${ }^{20}$ Th.De Donder, L'Affinité, Gauthier-Villars, Paris (1927)

${ }^{21}$ C.Stegelmann, A.Andreasen, C.T.Campbell, J.Am.Chem.Soc. 131 (2009) 8077

${ }^{22}$ J.A.Dumesic, G.W.Huber, M.Boudart, in Handbook of Heterogeneous Catalysis, ${ }^{\text {nd }}$ ed., G.Ertl, H.Knözinger, F.Schüth, J.Weitkamp, Eds., Wiley-VCH, Wienheim (2008), p.1445
} 
${ }^{23}$ J.X.Wang, N.M.Markovic, R.R.Adzic, J.Phys.Chem. 108 (2004) 4127

${ }^{24}$ S.Gottesfeld, in Fuel Cell Catalysis: A Surface Science Approach, M.T.M.Koper (Ed.), Wiley-VCH, Hoboken NJ, USA (2009) p.1

${ }^{25}$ D.van der Vliet, D.S. Strmenik, C.Wang, V.R.Stamenkovic, N.M.Markovic, M.T.M.Koper, J.Electroanal.Chem. 647 (2010) 29

${ }^{26}$ V.Stamenkovic, B.Fowler, B.S.Mun, G.Wang, P.N.Ross, C.A.Lucas, N.M.Markovic, Science 315 (2007) 493

${ }^{27}$ J.Greeley, I.E.L.Stephens, A.S.Bondarenko, T.P.Johanson, H.A.Hansen, T.F.Jaramillo, J.Rossmeisl, I.Chorkendorff, J.K.Norskov, Nature Chem. 1 (2009) 552 